http://dx.doi.org/10.32929/2446-8355.2020v29n2p232-255

\title{
MÉTODOS DE ESTIMATIVA DA EVAPOTRANSPIRAÇÃO DE REFERÊNCIA PARA O MUNICÍPIO DE IBIRUBÁ, RIO GRANDE DO SUL
}

\author{
Bruno Campos Mantovanelli ${ }^{1 *}$, Leonardo Chechi ${ }^{2}$, Ticiana François Magalhães ${ }^{3}$, Mirta \\ Teresinha Petry ${ }^{4}$, Lueni Gonçalves Terra ${ }^{3}$, Elenice Broetto Weiler ${ }^{5}$, Diego Dambrós \\ Londero $^{6}$, Juliano Dalcin Martins ${ }^{4}$
}

\footnotetext{
${ }^{1}$ Doutorando em Ciência do Solo, Física do solo, Universidade Federal de Santa Maria (UFSM), Santa Maria, RS. *E-mail do autor correspondente: brunomantovanelli21@gmail.com.

${ }^{2}$ Mestre em Engenharia Agrícola, Engenharia de água e solo, Universidade Federal de Santa Maria (UFSM), Santa Maria, RS.

${ }^{3}$ Doutoranda em Engenharia Agrícola, Engenharia de água e solo, Universidade Federal de Santa Maria (UFSM), Santa Maria, RS.

${ }^{4}$ Docente, Engenharia de água e solo, Universidade Federal de Santa Maria (UFSM), Santa Maria, RS.

${ }^{5}$ Doutoranda em Engenharia Florestal, Silvicultura, Universidade Federal de Santa Maria (UFSM), Santa Maria, RS.

${ }^{6}$ Engenheiro Agrônomo, Engenharia de água e solo, Universidade Federal de Santa Maria (UFSM), Santa Maria, RS.
}

Recebido: 11/02/2020; Aceito: 17/06/2020

RESUMO: O conhecimento preciso e a correta quantificação da evapotranspiração são essenciais para programas de irrigação e cálculo da demanda evaporativa da atmosfera. Objetiva-se com este trabalho comparar métodos de estimativa da evapotranspiração de referência para o município de Ibirubá-RS. Os dados utilizados correspondem às variáveis climatológicas no período de $1^{\circ}$ de outubro de 2017 a 31 de maio de 2018. Estas informações são constituídas pelas séries horárias de temperatura média (Tmed), mínima (Tmin) e máxima (Tmax), umidade relativa média (URmed), mínima (URmin) e máxima (URmax), velocidade média dos ventos a 2 metros de altura (u2), temperatura média do ponto de orvalho (Td) e radiação solar incidente $(\mathrm{K} \downarrow)$. Os métodos de determinação da evapotranspiração de referência (ETo) utilizados no trabalho foram Penman-Monteith (FAO-56), Penman, Priestley \& Taylor, Tanner \& Pelton, Makkink, Jensen-Haise, Hargreaves-Samani, Camargo, Benevides-Lopes, Turc e Linacre. Para fins de comparação, adotou-se o método de PenmanMonteith como método padrão para a estimativa ETo, comparando-se a os valores obtidos pelos demais métodos. Foram realizadas análises estatísticas de regressão, correlação e multivariada como critério de sumarização dos dados. Os resultados expressos a partir da análise de correlação e dos componentes principais, evidenciaram de forma adequada os reais efeitos das variáveis climatológicas sobre os métodos de estimativa testados. O efeito da radiação solar $\left(\mathrm{Q}^{*}\right)$ tem maior impacto sobre os métodos de estimativas de ETo.

Palavras-chave: Penman-Monteith (FAO-56). Penman. Radiação solar.

\section{REFERENCE EVAPOTRANSPIRATION ESTIMATION METHODS FOR THE CITY OF IBIRUBÁ, RIO GRANDE DO SUL}


ABSTRACT: The objective of this work is to compare methods of estimation of reference evapotranspiration for the municipality of Ibirubá-RS. The data used correspond to the climatological variables from October 1, 2017 to May 31, 2018. This information consists of hourly series of average (Tmed), minimum (Tmin) and maximum ( $\mathrm{T}$ max), average relative humidity (UR), minimum (URmin) and maximum (URmax), average wind speed at 2 meters high (u2), mean dew point temperature (Td) and incident solar radiation $(\mathrm{K} \downarrow)$. The methods for determining the reference evapotranspiration (ETo) used in the study were PenmanMonteith (FAO-56), Penman, Priestley \& Taylor, Tanner \& Pelton, Makkink, Jensen-Haise, Hargreaves-Samani, Camargo, Benevides-Lopes, Turc and Linacre. For comparison purposes, the Penman-Monteith method was adopted as the standard method for the ETo estimation, comparing to the values obtained by the other methods. Statistical regression, correlation and multivariate analyzes were performed as data summarization criteria. The results expressed from the analysis of correlation and main components, adequately evidenced the real effects of the climatological variables on the methods of estimation tested. The effect of solar radiation $\left(\mathrm{Q}^{*}\right)$ has a greater impact on ETo estimation methods.

Key words: Penman-Monteith (FAO-56). Penman. Solar radiation.

\section{INTRODUÇÃO}

O conhecimento preciso e a correta quantificação da evapotranspiração são essenciais para programas de irrigação, estimativa da demanda evaporativa da atmosfera, políticas de recursos hídricos e balanço de água no solo (PANDEY et al., 2016). A evapotranspiração pode ser expressa como uma função contínua de quatro variáveis meteorológicas principais: radiação líquida, déficit de pressão de vapor d’água, temperatura do ar e resistência aerodinâmica e uma variável da vegetação que é a resistência do dossel (STOY et al., 2006). Li et al. (2018) encontraram recentemente em suas pesquisas que a variabilidade interanual da evapotranspiração foi principalmente atribuída a mudanças na taxa da condutância do dossel em comparação às alterações nos fatores climáticos.

Alguns métodos de estimativa da evapotranspiração derivam de processos físicos que governam o processo, mas a maioria é de base empírica e geralmente depende de correlações entre uma evapotranspiração de referência e variáveis meteorológicas (SHARMA, 1985).

O método de Penman-Monteith (FAO-56) proposto por Allen et al. (1998) ainda é o mais aplicado para estimativa da ETo, por ser o que melhor se ajusta a medições em condições climatológicas variáveis (WIDMOSER, 2009). Berengena \& Gavilan (2005) reportaram uma clara tendência desse método em subestimar a evapotranspiração em ambientes áridos e semiáridos, possivelmente por uma advecção de calor sensível atuando como uma fonte extra de energia disponível para a evapotranspiração.

Há, assim, uma necessidade de estudos que avaliem métodos alternativos de estimativa da evapotranspiração, em especial os que necessitem de um menor número de informações meteorológicas e se ajustem às diferenças de clima para cada região. Por outro lado, a acurácia pode ser consideravelmente reduzida quando estes modelos são utilizados fora da faixa de calibração, o que significa que os ajustes precisam ser na maioria das vezes, locais 
(BERENGENA; GAVILAN, 2005). Diversos estudos já apontam a utilização de métodos mais simples e relatam a comparação de diferentes métodos na busca de resultados mais precisos da evapotranspiração (PANDEY et al. 2016; YU et al. 2016; ČADRO et al. 2017; LI et al. 2018).

Dado o empirismo característico de métodos com baixo requerimento de dados de entrada, verificações e calibrações regionais, quando não locais, propiciarão ganhos em termos qualitativos da estimativa da ETo e, consequente estabelecimento do manejo de irrigação. Diante do exposto, objetiva-se com este trabalho: (I) identificar o efeito e a correlação das variáveis climatológicas na estimativa da ETo nos diferentes métodos utilizados; (II) identificar a relação de similaridade entre métodos, quanto à explicação da variância total a partir das variáveis climatológicas; (III) simular diferentes cenários a partir dos dados climatológicos e quantificar seus efeitos residuais nas estimativas da ETo para os diferentes métodos.

\section{MATERIAL E MÉTODOS}

Os dados utilizados neste trabalho correspondem às variáveis climatológicas do município de Ibirubá-RS, no período de $1^{\circ}$ de outubro de 2017 a 31 de maio de 2018, correspondendo a oito meses sequenciais de dados, e foram obtidos junto ao Instituto Nacional de Meteorologia (INMET) disponíveis no endereço eletrônico (http://www.inmet.gov.br/). O curto período de avaliação de apenas 8 meses, se deve ao fator de melhor elucidar o comportamento das variáveis climatológicas e seus efeitos e impactos nos métodos de estimativas aplicados.

Estas informações são constituídas pelas séries horárias de temperatura média (Tmed), mínima (Tmin) e máxima (Tmax), umidade relativa média (URmed), mínima (URmin) e máxima (URmax), velocidade média dos ventos a 2 metros de altura (u2), temperatura média do ponto de orvalho $(\mathrm{Td})$ e radiação solar incidente $(\mathrm{K} \downarrow)$.

O município de Ibirubá localiza-se na região do Planalto Médio do Rio Grande do Sul, com altitude de $455 \mathrm{~m}$. O clima do local é Cfa conforme classificação estabelecida por Köppen onde a temperatura no mês mais quente é superior a $22^{\circ} \mathrm{C}$ e inferior a $18^{\circ} \mathrm{C}$ no mês mais frio, além de chuvas bem distribuídas ao longo do ano (KUINCHTNER; BURIOL, 2001).

A partir das séries horárias calculou-se a média diária das variáveis Tmed, Tmin, Tmax, Td, URmin, URmax, URmed, u2 e o somatório de $\mathrm{K} \downarrow$, descontando-se os valores noturnos negativos. Posteriormente, calculou-se o déficit de pressão de vapor (es-e), a radiação de energia incidente no topo da atmosfera $(\mathrm{K} 0 \downarrow)$ e o balanço de radiação $\left(\mathrm{Q}^{*}\right)$. Na Figura 1, é apresentada a variação destas variáveis ao longo do período analisado, em uma escala diária.

Os métodos de determinação da evapotranspiração de referência (ETo) utilizados no trabalho foram Penman-Monteith (FAO-56), Penman, Priestley \& Taylor, Tanner \& Pelton, Makkink, Jensen-Haise, Hargreaves-Samani, Camargo, Benevides-Lopes, Turc e Linacre. 
Para fins de comparação, adotou-se o método de Penman-Monteith como método padrão para a estimativa ETo, comparando-se a os valores obtidos pelos demais métodos.
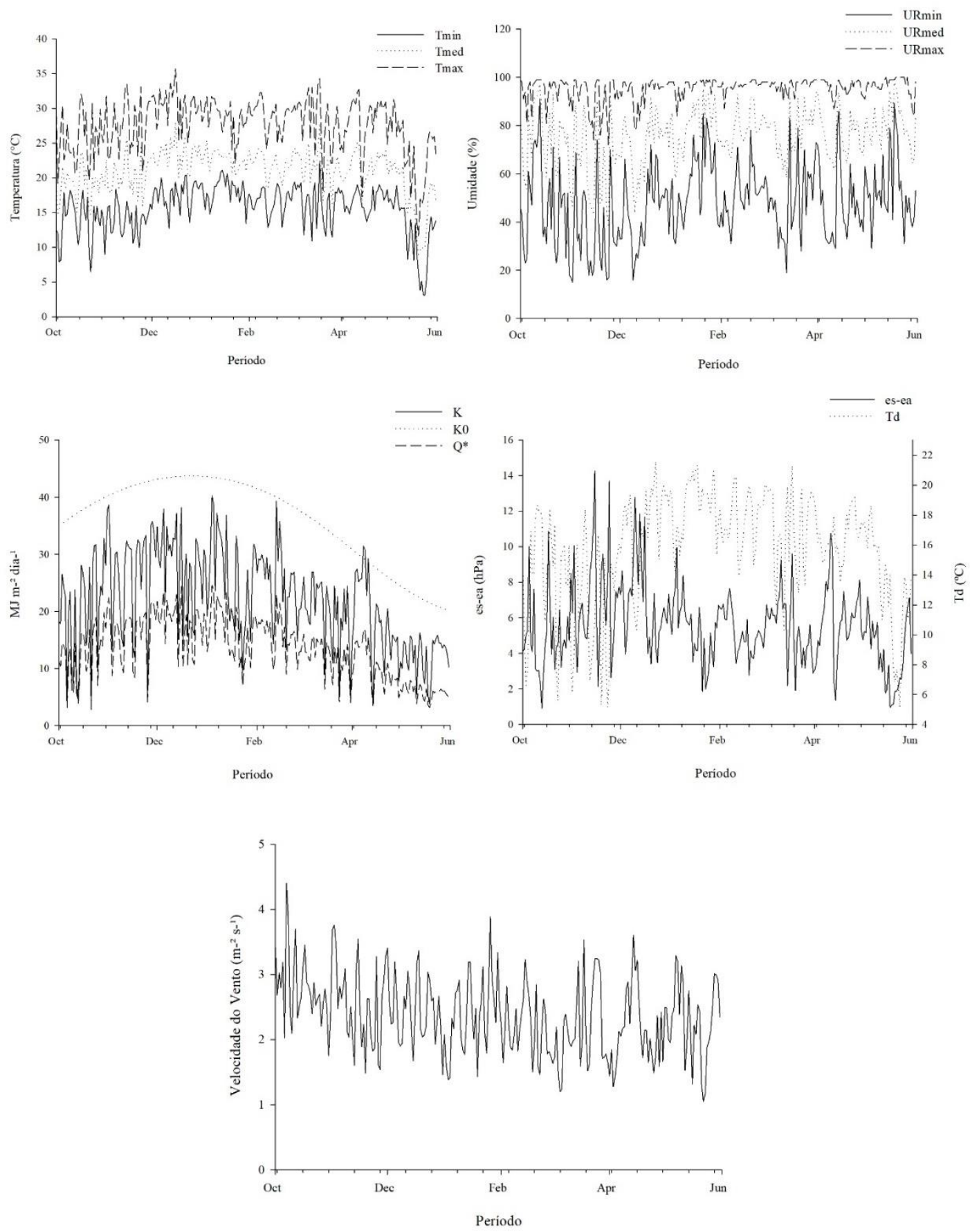

Figura 1. Comportamento das variáveis meteorológicas durante o período de outubro de 2017 a maio de 2018, no município de Ibirubá-RS. Behavior of meteorological variables during the period from October 2017 to May 2018, in the municipality of Ibirubá-RS.

Fonte: Autoria própria. Own authorship.

Primeiramente, realizou-se uma análise de regressão linear simples entre o método Penman-Monteith e os demais métodos, a fim de estabelecer o coeficiente de regressão (b) e determinação $\left(\mathrm{R}^{2}\right)$. Posteriormente, testou-se as hipóteses, hipóteses $\mathrm{H} 0: \mathrm{a}=0$ versus H1: $\mathrm{a} \neq$ 0 e $\mathrm{H} 0: b=1$ versus $\mathrm{H} 1: \mathrm{b} \neq 1$, por meio do teste $\mathrm{t}$ de Student a $5 \%$ de probabilidade de erro. 
Utilizou-se como indicador estatístico de qualidade dos métodos, a raiz do quadrado médio do erro (RMSE), calculado entre o método padrão e os demais métodos, conforme equação 1.

$$
\text { RMSE }=\sqrt{\frac{\sum_{i=1}^{\mathrm{n}}\left(\mathrm{T}_{\mathrm{i}}-\mathrm{Y}_{\mathrm{i}}\right)^{2}}{\mathrm{n}}}
$$

em que: Yi são os valores de ETo estimados pelo método de Penman-Monteith, Ŷi são os valores estimados pelos demais métodos.

Para avaliar a acurácia dos métodos de estimativa da ETo em diferentes condições climáticas é apresentado o gráfico dos resíduos de cada método em função das variáveis meteorológicas: u2, URmin, Tmed e $\mathrm{Q}^{*}$, tendo os resíduos calculados por (Yi) ^- Yi. Com base nas mesmas variáveis climáticas, dividiu-se o conjunto de dados em diferentes períodos, sendo, $1^{\circ}$ critério: dias com u $2<3 \mathrm{~m} \mathrm{~s}^{-1}$ e u $2>3 \mathrm{~m} \mathrm{~s}^{-1}, 2^{\circ}$ critério: dias com $\mathrm{Q}^{*}<\mathrm{Q}^{*}$ médio e dias com $\mathrm{Q}^{*}>\mathrm{Q}^{*}$ médio, $3^{\circ}$ critério: dias com Tmed $<$ Tmed médio e Tmed $>$ Tmed médio, e $4^{\circ}$ critério: dias com URmin $<45 \%$ e dias com URmin $>45 \%$. Para os diferentes períodos analisou-se os indicadores estatísticos $b, \mathrm{R}^{2}$, Test t e RMSE, conforme descrito acima.

Com a intenção de agrupar as variáveis e métodos para um conjunto mais significativo (representado pelos componentes) e identificar quais variáveis pertencem a quais componentes e o quanto cada variável explica cada componente, foi feito o estudo da Análise de Componentes Principais (ACP). Dessa forma, o conjunto inicial de doze variáveis passou a ser caracterizado por duas novas variáveis latentes ortogonais, o que possibilitou sua localização em figuras bidimensionais (ordenação dos acessos por componentes principais), que são combinações lineares das variáveis originais criadas com os dois maiores autovalores da matriz de covariância dos dados (HAIR et al., 2005). Todas as análises estatísticas multivariadas foram processadas no software STATISTICA® versão 7.0 (STATSOFT, 2004).

\section{RESULTADOS E DISCUSSÃO}

A representação gráfica nos componentes principais (Figura 2) permitiram caracterizar as variáveis que mais discriminaram na formação e diferenciação mediante os métodos avaliados, ficando evidente o efeito inverso da umidade relativa (URmin, URmed, URmax) e praticamente a contribuição nula do vento, visto a sua proximidade do eixo central de baixa contribuição. Quanto ao percentual de variância explicado pelas CPs (CP1 e CP2) observa-se que a primeira e a segunda componente principal explicam aproximadamente $71,1 \%$ da variabilidade dos dados, sendo a CP1 com 62,43\% e CP2 com 15,65\%.

Os índices de correlação evidenciaram o efeito das variáveis climatológicas sobre os métodos de estimativa da evapotranspiração (Tabela 1). O balanço de radiação ( $\left.\mathrm{Q}^{*}\right)$ apresentou correlação positiva com os métodos aplicados, apresentando índices superiores a 0,6, no qual destaca-se: $\mathrm{Q}^{*} \mathrm{x}$ Priestley $\&$ Taylor $=1,00 ; \mathrm{Q}^{*} \mathrm{x}$ Tanner \& Pelton $=1,00 ; \mathrm{Q}^{*} \mathrm{x}$ Makkink $=0,98 ; \mathrm{Q}^{*} \mathrm{x}$ Jensen $\&$ Haise $=0,97 ; \mathrm{Q}^{*} \mathrm{x}$ Turc $=0,98$.

Quanto à temperatura (Tmax; Tmin; Tmed) observou-se que a Tmin apresentou correspondência razoável com os métodos de Benevides \& Lopes $(0,44)$ e Camargo $(0,54)$, e 
para os demais métodos o índice de correlação variou entre $-0,03$ a 0,2 . As demais subdivisões da temperatura (Tmax e Tmed), apresentaram índices de correlação entre 0,54 e 0,90 para Tmax enquanto, para a Tmed a variação manteve-se entre 0,39 e 0,87 entre os métodos. No geral, verificou-se comportamento semelhante entre os métodos avaliados e estas subdivisões da temperatura, evidenciando assim eficiência nas estimativas, de forma positiva.

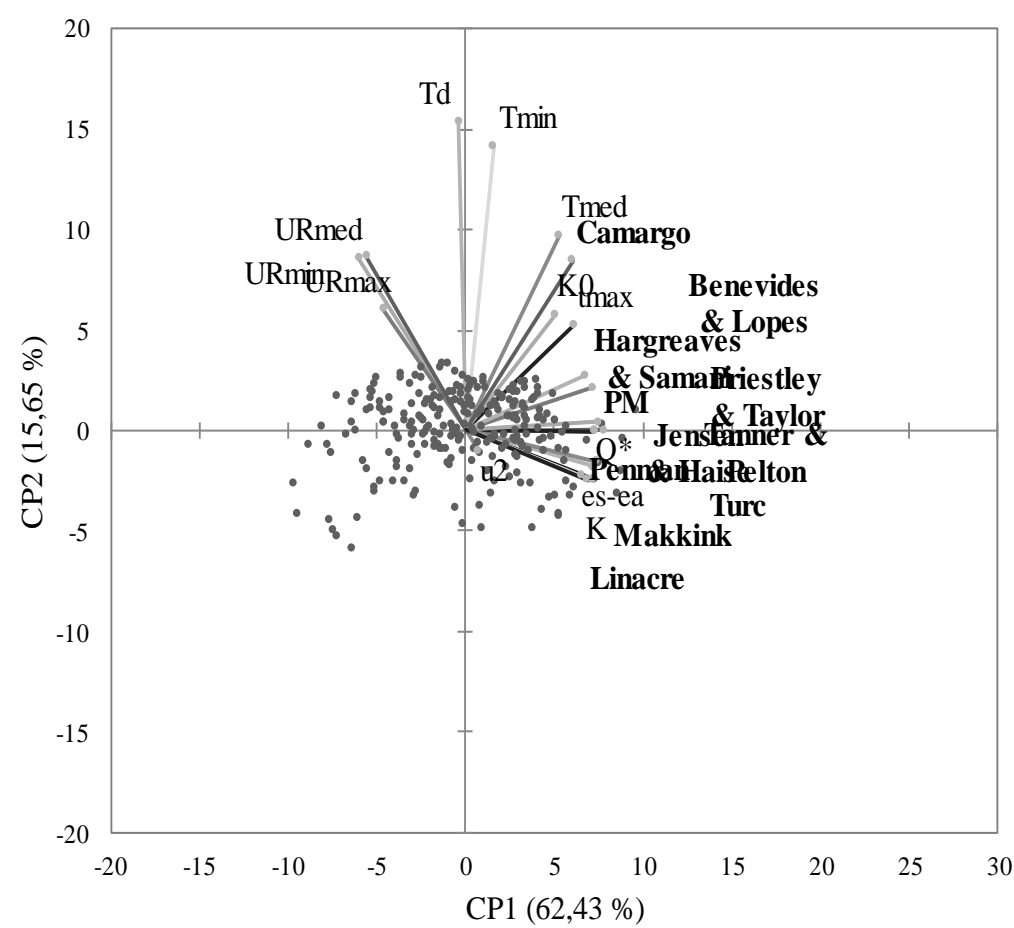

Figura 2. Componentes principais dos agrupamentos entre as variáveis climatológicas e os métodos de estimativa da ETo. Principal components of the clusters between climatological variables and ETo estimation methods.

Fonte: Autoria própria. Own authorship.

No que diz respeito à umidade relativa (URmax; URmin; URmed), houve correlação inversamente proporcional aos métodos aplicados. As subdivisões da umidade relativa são mais expressivas nos métodos de Penman $(-0,75 ;-0,73 ;-0,80)$ e Linacre $(-0,74 ;-0,80 ;-0,90)$ respectivamente a URmax, URmin e URmed. O método de Camargo apresentou correlações $(-0,27 ;-0,20 ;-0,28)$ respectivamente a URmax, URmin e URmed.

A semelhança evidenciada entre os métodos de estimativa da ETo a partir da ACP (Figura 2), pode ser explicada primeiramente pela alta variância explicada 78,1\% (CP1 e CP2), o que favorece um padrão de distribuição relativamente mais homogêneo, associando assim a uma maior semelhança entre os métodos, fato este, em função da sobreposição ocorrente na maioria dos métodos, mostrando assim a sua relação a partir das cargas fatoriais. Outra condição que é evidenciada a partir da ACP, é o fato das subdivisões da UR (URmin, URmed e URmax) apresentarem relação inversa aos métodos estudados, indicando que para Ibirubá, os efeitos da UR são preponderantes nas estimativas pelos métodos aplicados, sendo que, para futuras modificações nos métodos, a UR deve ser precisamente quantificada, para melhores estimativas. 
McVicar et al. (2012) constataram que a diminuição da evapotranspiração era globalmente generalizada por variáveis climatológicas, afetando de forma significativa os processos de estimativa da ETo, no qual observou-se que a correlação entre a ETo e radiação apresentavam coeficientes elevados superiores a 0,8. Em geral, o desempenho superior confirma que a Tmax e $\mathrm{Q}^{*}$ são os fatores dominantes para conduzir o processo de ETo, enquanto a velocidade do vento tem um impacto muito menor. Entretanto, destaca-se que a baixa contribuição dos índices de correlação do vento com os métodos, é evidente, pela grande parte dos métodos não os aplicar em suas equações, devido à grande variabilidade diária. $\mathrm{O}$ efeito do vento pode ser verificado a partir da análise de componentes principais, sendo sua contribuição, com os métodos, baixa e próximo à zona de distribuição fatorial (Figura 2).

Tabela 1. Valores das variáveis meteorológicas (VM) utilizadas para a determinação da evapotranspiração de referência e correlação linear de Pearson (CP) para os diferentes métodos. Meteorological variables (MV) used to determine the reference evapotranspiration and Pearson's linear correlation (CP) for the different methods.

\begin{tabular}{|c|c|c|c|c|c|c|c|c|c|c|c|}
\hline \multirow[b]{2}{*}{ Variáveis } & \multicolumn{11}{|c|}{ Métodos } \\
\hline & $\begin{array}{l}\text { Penman- } \\
\text { Monteith }\end{array}$ & Penman & $\begin{array}{c}\text { Priestley } \\
\& \\
\text { Taylor }\end{array}$ & $\begin{array}{l}\text { Tanner } \\
\& \\
\text { Pelton }\end{array}$ & Makkink & $\begin{array}{l}\text { Jensen } \\
\& \text { Haise }\end{array}$ & $\begin{array}{c}\text { Hargreaves } \\
\text { \& Samani }\end{array}$ & Camargo & $\begin{array}{c}\text { Benevides } \\
\text { \& Lopes }\end{array}$ & Turc & Linacre \\
\hline $\mathrm{K} 0 \downarrow$ & 0,67 & 0,46 & 0,71 & 0,71 & 0,58 & 0,61 & 0,79 & 0,90 & 0,41 & 0,59 & 0,35 \\
\hline $\mathrm{Q}^{*}$ & 0,97 & 0,74 & 1,00 & 1,00 & 0,98 & 0,97 & 0,85 & 0,73 & 0,63 & 0,98 & 0,64 \\
\hline Tmax & 0,70 & 0,74 & 0,57 & 0,58 & 0,54 & 0,69 & 0,77 & 0,68 & 0,90 & 0,62 & 0,78 \\
\hline Tmin & 0,18 & 0,17 & 0,10 & 0,10 & $-0,03$ & 0,18 & 0,20 & 0,59 & 0,44 & 0,04 & 0,14 \\
\hline Tmed & 0,61 & 0,63 & 0,48 & 0,48 & 0,39 & 0,60 & 0,65 & 0,77 & 0,87 & 0,48 & 0,66 \\
\hline URmax & $-0,52$ & $-0,75$ & $-0,36$ & $-0,36$ & $-0,40$ & $-0,43$ & $-0,44$ & $-0,27$ & $-0,61$ & $-0,42$ & $-0,74$ \\
\hline URmin & $-0,65$ & $-0,73$ & $-0,56$ & $-0,56$ & $-0,65$ & $-0,60$ & $-0,57$ & $-0,20$ & $-0,61$ & $-0,66$ & $-0,80$ \\
\hline URmed & $-0,69$ & $-0,82$ & $-0,58$ & $-0,58$ & $-0,65$ & $-0,63$ & $-0,61$ & $-0,28$ & $-0,71$ & $-0,66$ & $-0,90$ \\
\hline $\mathrm{Td}$ & $-0,05$ & $-0,14$ & $-0,06$ & $-0,06$ & $-0,19$ & 0,00 & 0,05 & 0,42 & 0,16 & $-0,13$ & $-0,19$ \\
\hline $\mathrm{u} 2$ & 0,09 & 0,31 & 0,05 & 0,05 & 0,03 & 0,02 & 0,09 & 0,07 & 0,07 & 0,03 & 0,09 \\
\hline es-e & 0,76 & 0,93 & 0,58 & 0,58 & 0,59 & 0,69 & 0,68 & 0,51 & 0,91 & 0,65 & 0,95 \\
\hline
\end{tabular}

Nota: Q*: Balanço de radiação; K0 $\downarrow$ : Radiação solar na ausência da atmosfera; U2: velocidade do vento; G: Densidade do fluxo de calor no solo; T: Temperatura do ar média diária; Tmáx: Temperatura máxima do ar; Tmín: Temperatura mínima do ar; Td: Temperatura do ponto de orvalho $\left({ }^{\circ} \mathrm{C}\right)$; d: Déficit de saturação do ar; UR: Umidade relativa do ar diária (\%). $Q^{*}$ : Radiation balance; K0 $\downarrow$ : Solar radiation in the absence of the atmosphere; U2: wind speed; G: Density of heat flow in the soil; T: Average daily air temperature; Tmax: Maximum air temperature; Tmin: Minimum air temperature; Td: Dew point temperature $\left({ }^{\circ} C\right)$; : Deficit of air saturation; $R H$ : Daily relative humidity (\%).

Fonte: Autoria própria. Own authorship.

Os índices de correlação encontrados para as variáveis climatológicas de radiação e temperatura são semelhantes aos obtidos por Lemos Filho et al. (2010); Tabari et al. (2011); Pandey et al. (2016), segundo os quais, métodos baseados nessas variáveis como Makkink, Tanner \& Pelton e Priestley \& Taylor, apresentaram as correspondências mais precisas de ETo (Tabela 1). Os métodos dependentes apenas da temperatura (Linacre) apresentaram relativamente baixa correlação, evidenciando a necessidade de modificações e calibrações conforme mudança extrema na temperatura. 
O efeito dos índices de correlação são evidentes em uma determinada região. Azevedo et al. (2003), estudando o efeito de variáveis climatológicas na ETo na região Centro Sul do Ceará, constataram que as variáveis climatológicas com maior representatividade nos processos da ETo foram a radiação e a velocidade do vento, enquanto que as temperaturas máximas e mínimas tiveram as menores influências nas estimativas, resultados contrários aos obtidos neste estudo. Isso evidencia a necessidade de implementação de um método específico em uma determinada região de estudo.

Os coeficientes de determinação foram superiores a 0,6 para os métodos aplicados, a exceção ao método de Camargo $\left(\mathrm{R}^{2}=0,575\right)$, enquanto o método de Jensen \& Haise $\left(\mathrm{R}^{2}=\right.$ 0,969) apresentou o maior coeficiente (Tabela 2 e Figura 3). Valores de RMSE estiveram abaixo de 1,0 para os métodos de Penman (RMSE = 0,760); Hargreaves \& Samani (RMSE = $0,929)$ e Linacre (RMSE $=0,928)$, métodos esses que apresentaram $\mathrm{R}^{2}$ razoavelmente bons (0,634 a 0,768), mas com RMSE baixos, evidenciando assim que apenas o $\mathrm{R}^{2}$ não é um bom estimador para a aceitação e confiabilidade nos métodos de estimativa da ETo. Efeito contrário observa-se para os métodos de Tanner \& Pelton $\left(\mathrm{R}^{2}=0,932\right.$; RMSE $\left.=2,794\right)$; Jensen \& Haise $\left(\mathrm{R}^{2}=0,969 ; \mathrm{RMSE}=1,907\right)$ e Makkink $\left(\mathrm{R}^{2}=0,897 ; \mathrm{RMSE}=2,498\right)$.

A partir do teste $t$ student (Tabela 2), os métodos de Penman ( $p=0,224)$ e Hargreaves \& Samani $(\mathrm{p}=0,473)$ não evidenciaram diferenças significativas quando comparado ao método padrão. Os demais métodos apresentaram efeito significativo $(\mathrm{p}<0,05)$ comparado ao método de Penman-Monteith. O coeficiente angular das equações de regressão (b) apresenta valores variando de 0,477 (Camargo) a 1,604 (Jensen \& Haise), podendo assim observar os métodos com a maior tendência em subestimar ou superestimar as medias a partir dos dados observados.

Tabela 2. Índices estatísticos para a comparação entre os métodos de estimativa da ETo em relação ao método Penman-Monteith. Statistical indexes for the comparison between ETo estimation methods in relation to the Penman-Monteith method.

\begin{tabular}{lcccc}
\hline \multicolumn{1}{c}{ Métodos } & $\mathrm{b}$ & $\mathrm{R}^{2}$ & $\mathrm{RMSE}$ & $\mathrm{t}$ Student \\
\hline Penman & 0,874 & 0,768 & 0,760 & $0,224^{\mathrm{ns}}$ \\
Priestley \& Taylor & 0,851 & 0,931 & 1,080 & $<0,001^{*}$ \\
Tanner \& Pelton & 1,573 & 0,932 & 2,794 & $<0,0001^{*}$ \\
Makkink & 1,422 & 0,897 & 2,498 & $<0,0001^{*}$ \\
Jensen \& Haise & 1,604 & 0,969 & 1,907 & $<0,0001^{*}$ \\
Hargreaves \& Samani & 0,851 & 0,795 & 0,929 & $0,473^{\mathrm{ns}}$ \\
Camargo & 0,477 & 0,575 & 1,279 & $<0,0001^{*}$ \\
Benevides\& Lopes & 0,620 & 0,617 & 1,095 & $0,0000^{*}$ \\
Turc & 1,200 & 0,942 & 1,092 & $<0,0001^{*}$ \\
Linacre & 0,534 & 0,634 & 0,928 & $<0,0001^{*}$ \\
\hline
\end{tabular}

Nota: $\mathrm{b}=$ coeficiente angular de regressão; $\mathrm{R}^{2}=$ Coeficiente de determinação; RMSE = raiz quadrada do erro quadrático médio. $b=$ slope of regression; $R^{2}=$ coefficient of determination; $R M S E=$ Root mean squared error . Fonte: Autoria própria. Own authorship. 

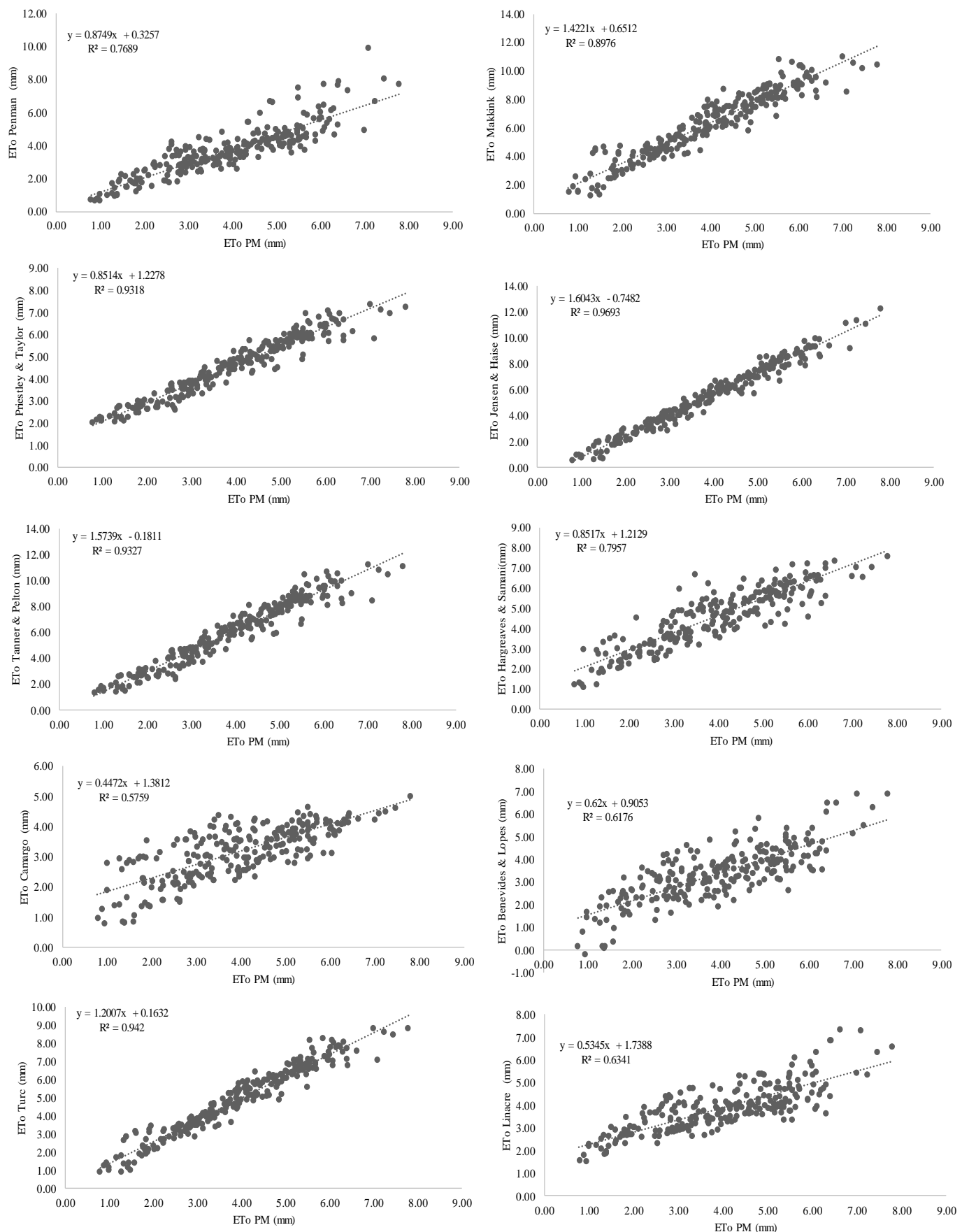

Figura 3. Regressão linear simples entre os diferentes métodos de estimativa da ETo e o método padrão para todo o período considerado. Simple linear regression between the different ETo estimation methods and the standard method for the entire period considered. Fonte: Autoria própria. Own authorship.

Dos métodos que estimam a ETo a partir da temperatura, Hargreaves \& Samani apresentou bom desempenho, visto que seus coeficientes estatísticos, assumindo os valores de RMSE e $\mathrm{R}^{2}$, são satisfatórios, e não diferiram estatisticamente do método padrão. Esse resultado está em desacordo com Conceição (2003), que afirma que métodos de estimativa de ETo com aplicação apenas da temperatura do ar como variável climática limitam a representatividade para efeito de estimativas da ETo, já que, conforme as condições de 
umidade e vento, a demanda hídrica atmosférica será diferente para os mesmos valores de temperatura do ar.

Silva et al. (2011), avaliando métodos de estimativa da Eto, também obtiveram bom desempenho para o método de Hargreaves \& Samani, com $\mathrm{R}^{2}=0,684$ e RMSE $=0,854$. O método de Hargreaves \& Samani é bem menos complexo que o de Penman-Monteith, podendo ser uma boa opção quando não existem dados de radiação solar global incidente, umidade relativa do ar e velocidade do vento (FERNANDES et al., 2010).

Os métodos de piores estimativas da Eto, como Tanner \& Pelton, Makkink e Jensen \& Haise, considerando seus valores de RMSE, são comumente citados na literatura, e foram utilizados por Borges Júnior et al. (2012) em Garanhuns-PE e Hallal et al. (2017) em PelotasRS. Apesar do método Makkink ser elaborado a partir do método de Penman, entretanto, apresenta bons resultados em climas muito úmidos, fato não evidenciado para Ibirubá, enquanto que o de Jensen \& Haise foi desenvolvido para regiões áridas e semiáridas, condição também não encontrada em Ibirubá.

O relativo bom desempenho do método Linacre, a partir do RMSE contrastante com o método padrão, é decorrente na literatura em função de ser baseado em uma simplificação da metodologia Penman-Monteith (FERNANDES et al., 2010). O satisfatório desempenho do método Priestley \& Taylor foi evidente, por se assemelhar ao método padrão, exigindo praticamente os mesmos elementos climatológicos; porém, sua aplicação acaba sendo restringida para locais onde não há carência de equipamentos para medi-los (SILVA et al., 2011).

O coeficiente angular das equações de regressão (b) (Tabela 2) e as variações da ETo para os diferentes métodos (Figura 4), evidenciam a variação e a amplitude relacionadas aos aspectos da subestimação e superestimação para os métodos aplicados, quando comparados ao método padrão, tornando notório os efeitos das diferentes estações que abrangem os dados (primavera, verão e outono), e as exigências climáticas dos métodos avaliados. As maiores amplitudes de superestimação ficam evidentes para os métodos de Makkink (58,52\%), Tanner \& Pelton $(52,67 \%)$, Jensen \& Haise $(41,22)$, Turc $(24,17)$, Priestley \& Taylor $(16,2 \%)$ e Hargreaves \& Samani (16\%), enquanto que, o método de Camargo subestimou em $(20,10 \%)$, Benevides \& Lopes (15,01\%), Penman (12,6\%) e Linacre $(2,29 \%)$.

O elevado desvio da ETo estimado pelas equações de Makkink, Tanner \& Pelton e Jensen \& Haise pode ser esperado quando esses métodos são extrapolados para outras áreas climáticas sem recalibrar as constantes envolvidas nas fórmulas. Ou seja, a escolha da equação de ETo por esses métodos, deve priorizar a origem e as condições ambientais de seu desenvolvimento.

O modelo de Hargreaves \& Samani, destacado como de bom desempenho, corrobora com resultados de Lima Júnior et al. (2016), que constataram uma superestimação abaixo de $20 \%$ para o método, tornando válida a informação de que esse tende a superestimar a ETo em regiões úmidas e subestimá-lo em regiões muitos secas e em regiões de velocidade de vento superiores a $4 \mathrm{~ms}^{-1}$ (TEMESGEN et al., 1999). 

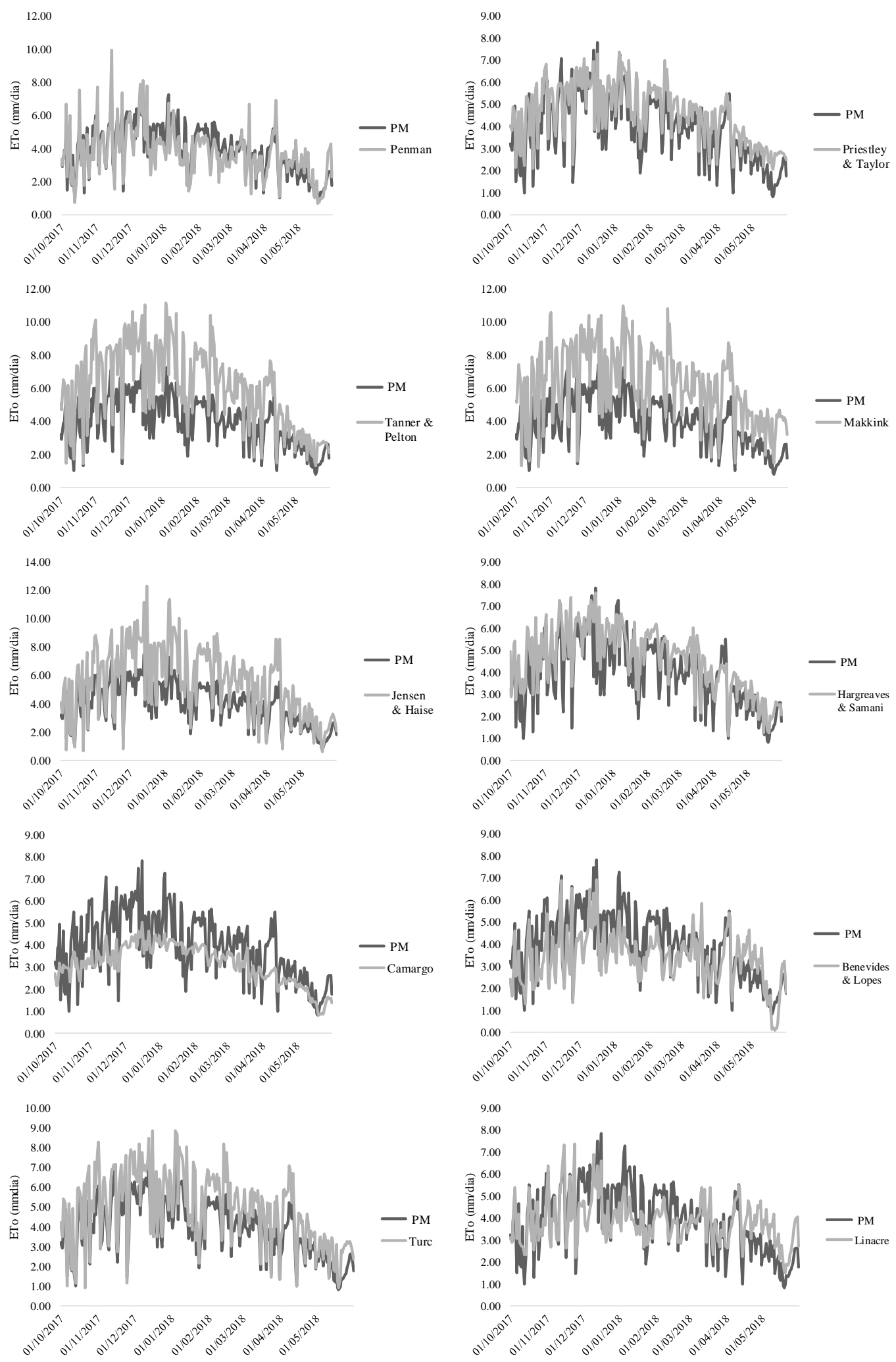

Figura 4. Comparação dos valores ETo a partir do método padrão com estimativas realizadas por métodos empíricos e valores de variações de subestimação e superestimação. Comparison of ETo values from the standard method and estimates by methods and variations of underestimation and overestimation.

Fonte: Autoria própria. Own authorship. 
A equação de Penman subestimou a ETo em aproximadamente 13\%, mas ainda assim, produz boas estimativas de ETo. No campo da inferência, e conforme destacado por Ahmed e Hussein (1999), o método pode ser usado com precisão no lugar da equação de PenmanMonteith e seu desempenho é significativamente melhorado quando usado com déficits de pressão de vapor de saturação calculados usando as temperaturas máximas e mínimas do ar.

Com exceção dos métodos de Penman e Penman-Monteith (PM), que são métodos semi-analíticos de estimativa da ETo, os diferentes métodos utilizam relações empíricas com diferentes variáveis meteorológicas correlacionadas. Dessa forma, na Tabela 3 e Figura 5, são apresentados os resíduos das estimativas entre a ETo estimada pelo método padrão e os demais métodos, em função da variação na umidade relativa mínima (URmin).

Para Penman, é observado que, para condições de URmin baixa $(<45 \%)$, ocorre aumento no valor dos resíduos positivos $(2 \mathrm{~mm})$ e negativos $(-3 \mathrm{~mm})$. Por outro lado, os métodos Priestley \& Taylor e Turc apresentaram menor amplitude em seus resíduos, sendo de1,5 a 1,5 mm e de $-2,5$ a $0,5 \mathrm{~mm}$, respectivamente. Para ambos os métodos, a maior parte dos resíduos ficaram no campo negativo, também apresentando maior variação quando da URmin abaixo de $45 \%$.

O método Tanner \& Pelton apresentou a maior amplitude de resíduos, variando entre -6 e $2 \mathrm{~mm}$, apresentando distribuição uniforme nas diferentes condições de URmin. Os métodos Makkink, Jensen \& Haise, Hargreaves \& Samani e Camargo exibiram uma faixa de amplitude dos resíduos de $5 \mathrm{~mm}$, sendo de -5 a $0 \mathrm{~mm}$, de -4 a $1 \mathrm{~mm}$, de $-3,5$ a $1,5 \mathrm{~mm}$ e de -2 a $3 \mathrm{~mm}$, respectivamente. Para Makkink, Jensen \& Haise e Hargreaves \& Samani os resíduos mais negativos se apresentaram com URmin abaixo de 45\%, enquanto que para Camargo observaram-se desvios positivos para URmin abaixo de $45 \%$ e negativos para URmin acima de 60\%. Para Benevides \& Lopes e Linacre nota-se uma distribuição uniforme dos resíduos, com amplitude 4,5 (-2,5 - 3) e 4,8 (-1,8 - 3) mm, respectivamente.

Tabela 3. Índices estatísticos para a comparação entre métodos de estimativa da ETo para umidade relativa maior e menor que $45 \%$. Statistical indexes for comparing ETo estimation methods for relative humidity greater than and less than $45 \%$.

\begin{tabular}{lcccccccc}
\hline \multicolumn{1}{c}{ Métodos } & $\mathrm{b}$ & $\mathrm{R}^{2}$ & RMSE & $\mathrm{t}$ Student & $\mathrm{b}$ & $\mathrm{R}^{2}$ & RMSE & t Student \\
\hline Penman & -------- & URmin $<45 \%$ & ------- & --------- & URmin $>45 \%$ & -------- \\
Priestley \& Taylor & 0,839 & 0,648 & 0,840 & $0,859^{\text {ns }}$ & 0,749 & 0,772 & 0,687 & $0,070^{\text {ns }}$ \\
Tanner \& Pelton & 0,858 & 0,889 & 0,616 & $0,012^{*}$ & 0,909 & 0,954 & 0,866 & $<0,0001^{*}$ \\
Makkink & 1,588 & 0,892 & 2,971 & $<0,0001^{*}$ & 1,681 & 0,955 & 2,641 & $<0,0001^{*}$ \\
Jensen \& Haise & 1,315 & 0,834 & 2,794 & $<0,0001^{*}$ & 1,498 & 0,901 & 2,229 & $<0,0001^{*}$ \\
Hargreaves \& Samani & 1,590 & 0,948 & 2,218 & $<0,0001^{*}$ & 1,667 & 0,975 & 1,610 & $<0,0001^{*}$ \\
Camargo & 0,838 & 0,718 & 0,876 & $0,006^{*}$ & 0,874 & 0,784 & 0,971 & $<0,0001^{*}$ \\
Benevides \& Lopes & 0,542 & 0,718 & 1,623 & $<0,0001^{*}$ & 0,513 & 0,570 & 0,908 & $0,061^{\text {ns }}$ \\
Turc & 0,573 & 0,471 & 1,220 & $<0,0001^{*}$ & 0,555 & 0,571 & 0,982 & $0,001^{*}$ \\
Linacre & 1,119 & 0,905 & 1,227 & $<0,0001^{*}$ & 1,258 & 0,944 & 0,969 & $<0,0001^{*}$ \\
\hline
\end{tabular}

Fonte: Autoria própria. Own authorship. 

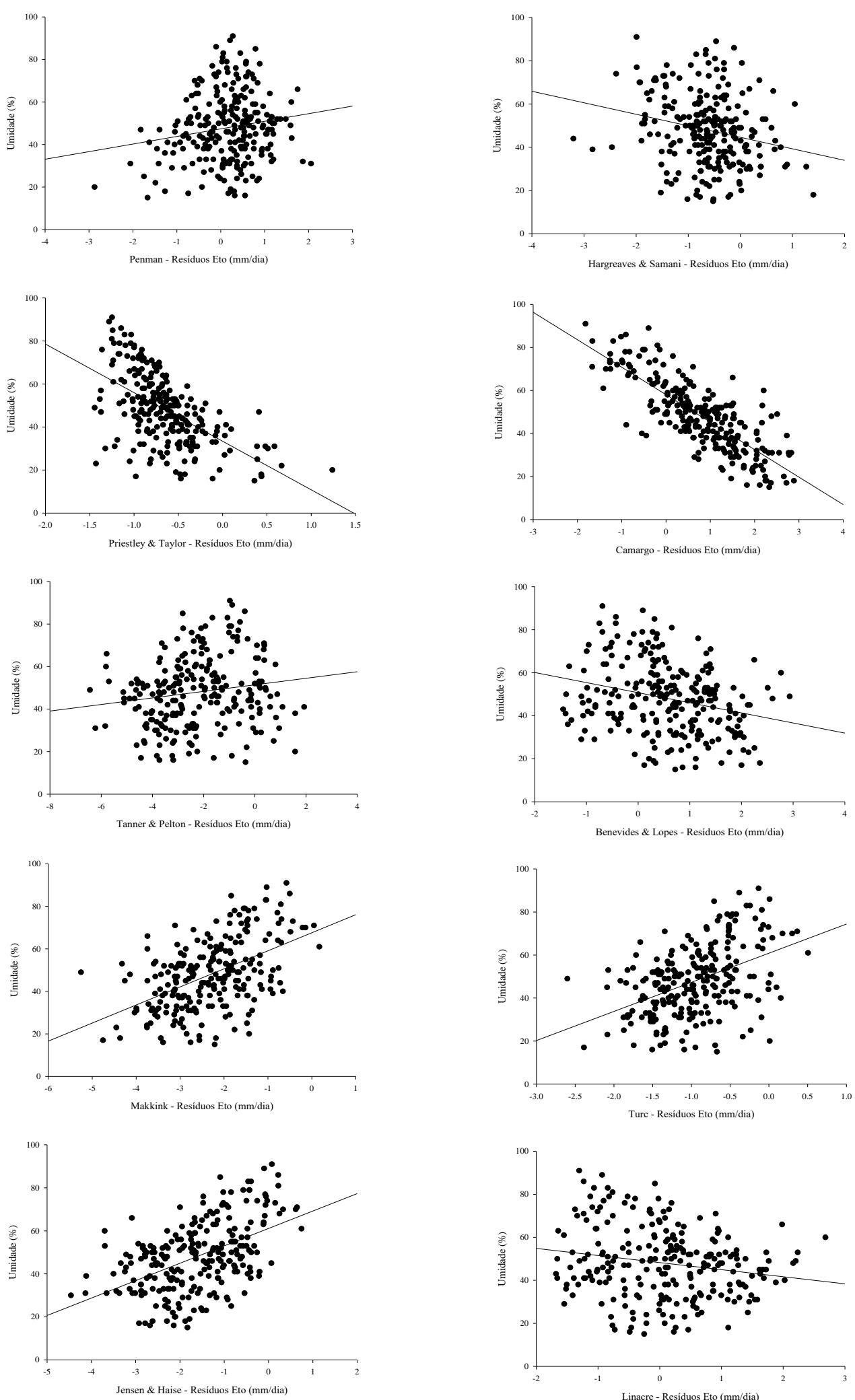

Figura 5. Resíduos da relação entre a variável umidade relativa mínima (URmin) e sua influência nos métodos de determinação da Eto. Figure 5 - Residues of the relationship between the minimum relative humidity (URmin) variable and its influence on Eto determination methods.

Fonte: Autoria própria. Own authorship. 
Na Tabela 3 são apresentados os índices estatísticos para a ETo estimados pelos diferentes métodos, dividindo-se em dois períodos de dias com URmin menor e maior que 45\%. Analisando o RMSE, todos os métodos apresentaram valores menores, considerando-se apenas os dias com URmin $>45 \%$, exceto o método de Priestley \& Taylor. O método de Camargo foi o que apresentou a maior diminuição no RMSE, decrescendo de 1,623 em condições de alta URmin para 0,908 mm em condições de baixa URmin.

$\mathrm{Na}$ Tabela 4 e Figura 6 são apresentados os resíduos da estimativa da ETo entre Penman-Monteith e os demais métodos em função do balanço de energia (Q*). É possível verificar uma distribuição relativamente uniforme dos resíduos, nas diferentes disponibilidades de $\mathrm{Q}^{*}$. Destacam-se valores de RMSE próximos de $3 \mathrm{~mm}$ para dias com $\mathrm{Q}^{*}$ $>20 \mathrm{MJ} \mathrm{m}^{-2}$ dia $^{-1}$ nos métodos de Benevides \& Lopes e Linacre. No entanto, os dados da Tabela 4 indicam que todos os métodos apresentam diminuição no RMSE quando $Q^{*}<$ média (13,36 $\mathrm{MJ} \mathrm{m}^{-2}$ dia $\left.^{-1}\right)$, com exceção de Priestley \& Taylor e Hargreaves \& Samani, para os quais se observa ligeiro aumento no valor do RMSE quando $\mathrm{Q}^{*}<$ média. Os métodos Tanner \& Pelton, Makkink e Jensen \& Haise que apresentaram os maiores valores de RMSE, mostram reduções de 2,072; 1,295 e 1,477 mm no valor do RMSE, respectivamente, quando $\mathrm{Q}^{*}<13,36 \mathrm{MJ} \mathrm{m}^{-2} \mathrm{dia}^{-1}$.

Tabela 4. Índices estatísticos para a comparação entre métodos de estimativa da ETo para Rn maior e menor que a média em relação ao método Penman-Monteith. Statistical indexes for the comparison between ETo estimation methods for Rn higher and lower than the average in relation to the Penman-Monteith method.

\begin{tabular}{|c|c|c|c|c|c|c|c|c|}
\hline Métodos & $\mathrm{b}$ & $\mathrm{R}^{2}$ & RMSE & t Student & $\mathrm{b}$ & $\mathrm{R}^{2}$ & RMSE & t Student \\
\hline & \multicolumn{4}{|c|}{---------- Q* < Média ---------- } & \multicolumn{4}{|c|}{---------- Q* > Média ---------- } \\
\hline Penman & 1,101 & 0,740 & 0,608 & $0,225 \mathrm{~ns}$ & 1,144 & 0,656 & 0,874 & $0,001 *$ \\
\hline Priestley \& Taylor & 0,741 & 0,844 & 0,827 & $<0,0001^{*}$ & 0,696 & 0,770 & 0,702 & $<0,0001 *$ \\
\hline Tanner \& Pelton & 1,374 & 0,843 & 1,504 & $<0,0001 *$ & 1,288 & 0,776 & 3,576 & $<0,0001 *$ \\
\hline Makkink & 1,212 & 0,735 & 1,731 & $<0,0001 *$ & 1,123 & 0,683 & 3,026 & $<0,0001 *$ \\
\hline Jensen \& Haise & 1,492 & 0,924 & 0,981 & $<0,0001 *$ & 1,531 & 0,902 & 2,458 & $<0,0001 *$ \\
\hline Hargreaves \& Samani & 1,041 & 0,656 & 1,088 & $<0,0001 *$ & 0,788 & 0,573 & 0,759 & $0,000 *$ \\
\hline Camargo & 0,626 & 0,407 & 0,748 & $0,774 \mathrm{~ns}$ & 0,429 & 0,424 & 1,613 & $<0,0001 *$ \\
\hline Benevides \& Lopes & 0,961 & 0,597 & 0,704 & $0,595 \mathrm{~ns}$ & 0,793 & 0,541 & 1,353 & $<0,0001 *$ \\
\hline Turc & 1,082 & 0,848 & 0,719 & $<0,0001 *$ & 1,011 & 0,822 & 1,342 & $<0,0001 *$ \\
\hline Linacre & 0,681 & 0,585 & 0,848 & $<0,0001 *$ & 0,719 & 0,499 & 0,994 & $<0,0001 *$ \\
\hline
\end{tabular}

Nota: $\mathrm{b}=$ coeficiente angular de regressão; $\mathrm{R}^{2}=$ Coeficiente de determinação; RMSE = raiz quadrada do erro quadrático médio. $b=$ slope of regression; $R^{2}=$ coefficient of determination; $R M S E=$ root mean squared error . Fonte: Autoria própria. Own authorship. 

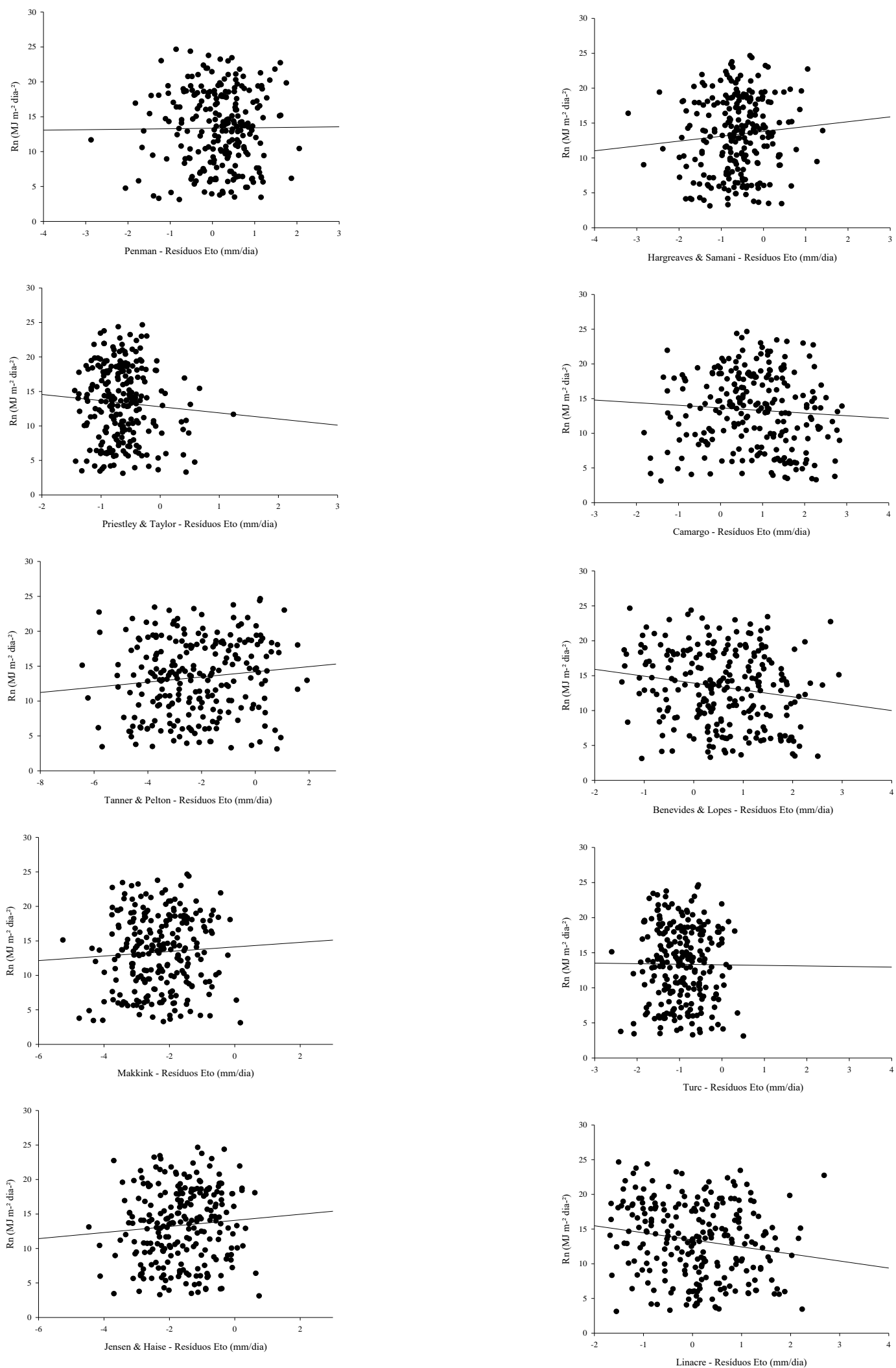

Figura 6. Resíduos da relação entre a variável balanço de radiação $\left(\mathrm{Q}^{*}\right)$ e sua influência nos métodos de determinação da ETo. Residues of the relationship between the variable radiation balance $\left(Q^{*}\right)$ and its influence on ETo determination methods.

Fonte: Autoria própria. Own authorship. 
A Tabela 5 e a Figura 7 apresentam os resíduos das estimativas entre a ETo pelo método padrão $(\mathrm{PM})$ e os demais métodos, em função da variação na temperatura média (Tmed). Para o método de Penman, observam-se desvios variando de -3 a $2 \mathrm{~mm}$, sendo que os maiores desvios ocorreram quando a temperatura média estava acima de $20^{\circ} \mathrm{C}$, corroborando com os dados da Tabela 5, na qual é possível observar que o valor de RMSE foi menor no subperíodo de dias com Tmed < média $\left(20,92^{\circ} \mathrm{C}\right)$.

Por outro lado, para Priestley \& Taylor, evidenciam-se desvios variando de -1,5 a 1,5, sendo que os maiores desvios aconteceram quando da ocorrência de dias com temperatura média do ar abaixo de $15{ }^{\circ} \mathrm{C}$ e acima de $25^{\circ} \mathrm{C}$. Na Tabela 5 também verifica-se que esse método apresenta o maior valor de RMSE para dias com Tmed < média $(0,939)$, quando comparado a dias com Tmed > média $(0,601)$.

Para Jensen \& Haise observam-se desvios negativos chegando a $-4 \mathrm{~mm}$, quando da ocorrência de altas temperaturas $\left(>20^{\circ} \mathrm{C}\right)$, e RMSE de $1,378 \mathrm{~mm}$ e 2,222 para Tmed menor e maior que a média, respectivamente. Os demais métodos não demonstraram variações significativas de RMSE, tendo os resíduos dispostos de maneira uniforme em função da variação da temperatura média do ar (Tabela 5 e Figura 6).

Tabela 5. Índices estatísticos para a comparação entre métodos de estimativa da ETo para temperatura maior e menor que a média. Statistical indexes for comparing ETo estimation methods for higher and lower than average temperatures.

\begin{tabular}{|c|c|c|c|c|c|c|c|c|}
\hline Métodos & $\mathrm{b}$ & $\mathrm{R}^{2}$ & RMSE & t Student & $\mathrm{b}$ & $\mathrm{R}^{2}$ & RMSE & t student \\
\hline & \multicolumn{4}{|c|}{------- Tmed < Média --------- } & \multicolumn{4}{|c|}{-------- Tmed > Média ---------- } \\
\hline Penman & 0,792 & 0,789 & 0,642 & $0,345^{\text {ns }}$ & 0,872 & 0,659 & 0,837 & $0,310^{\text {ns }}$ \\
\hline Priestley \& Taylor & 0,943 & 0,952 & 0,939 & $<0,0001^{*}$ & 0,856 & 0,912 & 0,601 & $0,002^{*}$ \\
\hline Tanner \& Pelton & 1,741 & 0,952 & 2,583 & $<0,0001^{*}$ & 1,583 & 0,913 & 2,942 & $<0,0001^{*}$ \\
\hline Makkink & 1,663 & 0,913 & 2,561 & $<0,0001^{*}$ & 1,446 & 0,916 & 2,451 & $<0,0001 *$ \\
\hline Jensen \& Haise & 1,563 & 0,964 & 1,378 & $<0,0001^{*}$ & 1,606 & 0,956 & 2,222 & $<0,0001^{*}$ \\
\hline Hargreaves \& Samani & 0,835 & 0,767 & 0,886 & $0,002^{*}$ & 0,757 & 0,707 & 0,961 & $<0,0001^{*}$ \\
\hline Camargo & 0,369 & 0,415 & 1,175 & $0,000^{*}$ & 0,364 & 0,487 & 1,351 & $<0,0001^{*}$ \\
\hline Benevides \& Lopes & 0,503 & 0,574 & 1,115 & $<0,0001^{*}$ & 0,452 & 0,457 & 1,080 & $<0,0001^{*}$ \\
\hline Turc & 1,329 & 0,944 & 1,126 & $<0,0001^{*}$ & 1,196 & 0,939 & 1,066 & $<0,0001^{*}$ \\
\hline Linacre & 0,506 & 0,684 & 0,825 & $0,345^{*}$ & 0,458 & 0,438 & 0,998 & $0,047^{*}$ \\
\hline
\end{tabular}

Nota: $\mathrm{b}=$ coeficiente angular de regressão; $\mathrm{R}^{2}=$ Coeficiente de determinação; RMSE = raiz quadrada do erro quadrático médio. $b=$ slope of regression; $R^{2}=$ coefficient of determination; $R M S E=$ Root mean squared error . Fonte: Autoria própria. Own authorship. 

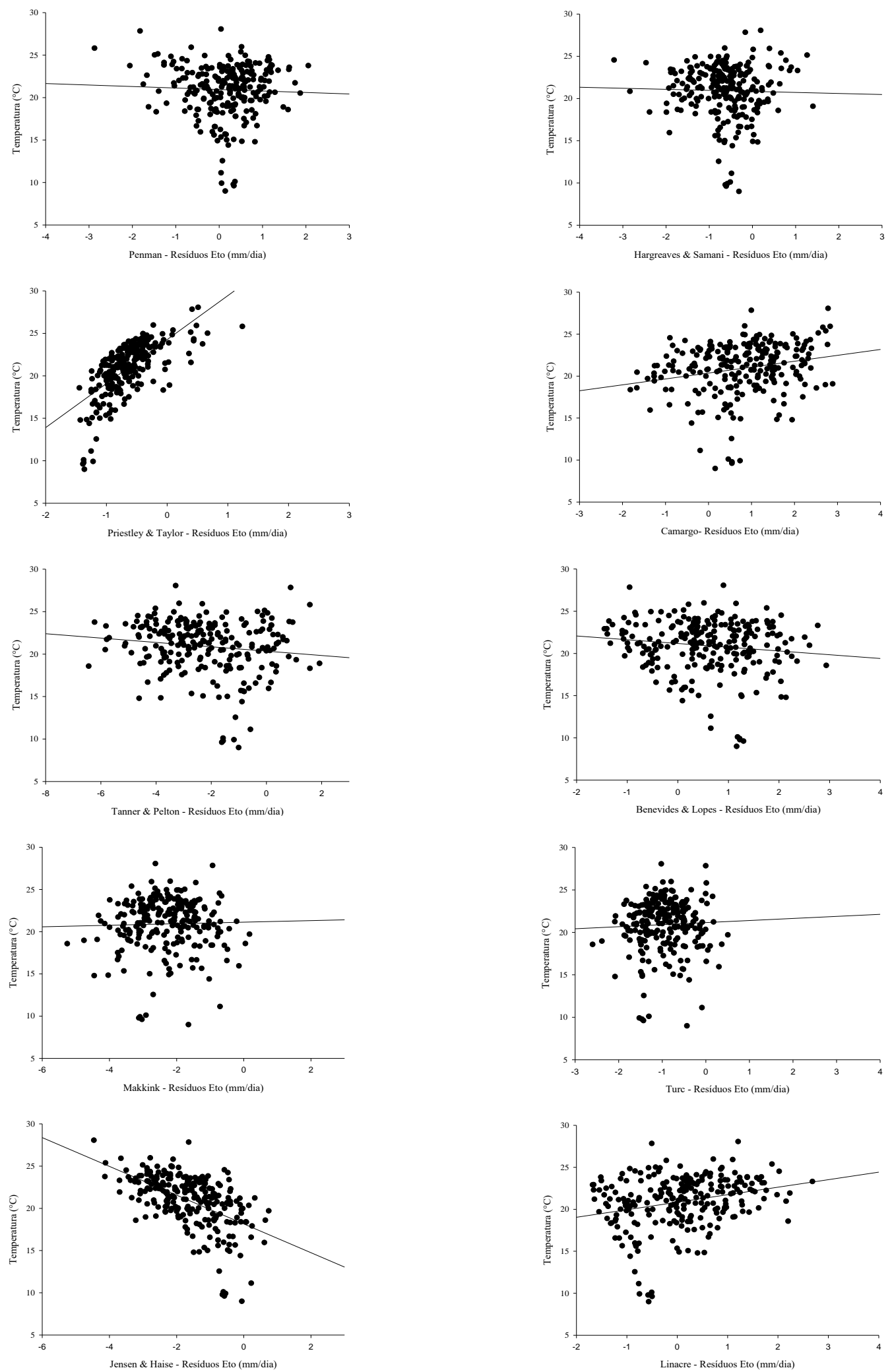

Figura 7. Resíduos da relação entre a variável temperatura média (Tmed) e sua influência nos métodos de determinação da ETo. Residues of the relationship between the mean temperature variable (Tmed) and its influence on ETo determination methods.

Fonte: Autoria própria. Own authorship. 
Os resíduos das estimativas entre a ETo estimada pelo método Penman-Monteith e os demais métodos em função da variação na velocidade média do vento a $2 \mathrm{~m}$ de altura $\left(\mathrm{u}_{2}\right)$ são visualizados na Tabela 6 e Figura 8. Observa-se que os resíduos das estimativas dos diferentes métodos apresentam-se distribuídos de maneira uniforme, com uma pequena tendência de resíduos negativos pelo método de Hargreaves \& Samani quando da ocorrência de vento acima de $3 \mathrm{~m} \mathrm{~s}^{-1}$.

De forma geral, quanto ao RMSE, observam-se valores menores para $\mathrm{u}_{2}<3 \mathrm{~m} \mathrm{~s}^{-1}$, para os métodos de Penman, Priestley \& Taylor, Camargo e Hargreaves \& Samani enquanto o contrário acontece para os métodos Tanner \& Pelton, Makkink, Jensen \& Haise, Benevides \& Lopes, Turc e Linacre.

Tabela 6. Índices estatísticos para a comparação entre métodos de estimativa da ETo para vento maior e menor que 3,0 $\mathrm{m} \mathrm{s}^{-1}$ em relação ao método Penman-Monteith. Statistical indexes for the comparison between ETo estimation methods for wind greater and less than $3.0 \mathrm{~m} \mathrm{~s}^{-1}$ in relation to the Penman-Monteith method.

\begin{tabular}{|c|c|c|c|c|c|c|c|c|}
\hline Métodos & $\mathrm{b}$ & $\mathrm{R}^{2}$ & RMSE & t Student & $\mathrm{b}$ & $\mathrm{R}^{2}$ & RMSE & t Student \\
\hline & \multicolumn{4}{|c|}{---- $\mathrm{u}_{2}<3 \mathrm{~m} \mathrm{~s}^{-1}$} & \multicolumn{4}{|c|}{------ $\mathrm{u}_{2}>3 \mathrm{~m} \mathrm{~s}^{-1}$------ } \\
\hline Penman & 0,824 & 0,803 & 0,723 & $0,240^{\mathrm{ns}}$ & 1,113 & 0,813 & 0,913 & $0,183^{*}$ \\
\hline Priestley \& Taylor & 0,862 & 0,946 & 0,762 & $0,090^{\mathrm{ns}}$ & 0,797 & 0,870 & 0,770 & $0,320^{*}$ \\
\hline Tanner \& Pelton & 1,594 & 0,946 & 2,894 & $<0,0001^{*}$ & 1,474 & 0,872 & 2,252 & $0,004^{*}$ \\
\hline Makkink & 1,428 & 0,909 & 2,528 & $<0,0001^{*}$ & 1,392 & 0,852 & 2,354 & $0,010^{*}$ \\
\hline Jensen \& Haise & 1,621 & 0,975 & 1,949 & $<0,0001^{*}$ & 1,522 & 0,949 & 1,690 & $0,005^{*}$ \\
\hline Hargreaves \& Samani & 0,845 & 0,800 & 0,920 & $0,424^{\mathrm{ns}}$ & 0,882 & 0,776 & 0,971 & $0,994^{*}$ \\
\hline Camargo & 0,466 & 0,600 & 1,262 & $<0,0001^{*}$ & 0,355 & 0,459 & 1,355 & $<0,0001^{\mathrm{n}}$ \\
\hline Benevides \& Lopes & 0,611 & 0,608 & 1,110 & $0,001^{*}$ & 0,661 & 0,661 & 1,018 & $0,189^{*}$ \\
\hline Turc & 1,200 & 0,951 & 1,110 & $0,003^{*}$ & 1,177 & 0,906 & 1,005 & $0,177^{*}$ \\
\hline Linacre & 0,509 & 0,623 & 0,949 & $<0,0001^{*}$ & 0,653 & 0,700 & 0,821 & $0,117^{*}$ \\
\hline
\end{tabular}

Nota: $\mathrm{b}=$ coeficiente angular de regressão; $\mathrm{R}^{2}=$ Coeficiente de determinação; RMSE = raiz quadrada do erro quadrático médio. $b=$ slope of regression; $R^{2}=$ coefficient of determination; $R M S E=$ Root mean squared error . Fonte: Autoria própria. Own authorship.

Os métodos que apresentaram maior acurácia, quando comparados com o método de Penman-Monteith, considerando o RMSE, foram em ordem decrescente: Penman, Linacre, Hargreaves \& Samani, Priestley \& Taylor, Turc, Benevides \& Lopes, Camargo, Jensen \& Haise, Makkink e Tanner \& Pelton. Observando-se a significância do teste $\mathrm{t}$ de student ( $\mathrm{p}<0,005)$, apenas os métodos de Penman e Hargreaves \& Samani não apresentaram diferença estatística do método padrão.

Analisando os métodos em diferentes condições climatológicas, é possível observar que, em determinadas situações, alguns métodos apresentam melhorias nas estimativas da ETo. Penman, por se tratar de um método semi-analítico, em que considera o balanço de radiação, o déficit de saturação (temperatura e umidade) e a velocidade dos ventos, engloba todas as variáveis meteorológicas que influenciam na ETo, não apresentando grande variação no erro de suas estimativas quando da ocorrência de diferentes condições atmosféricas. 

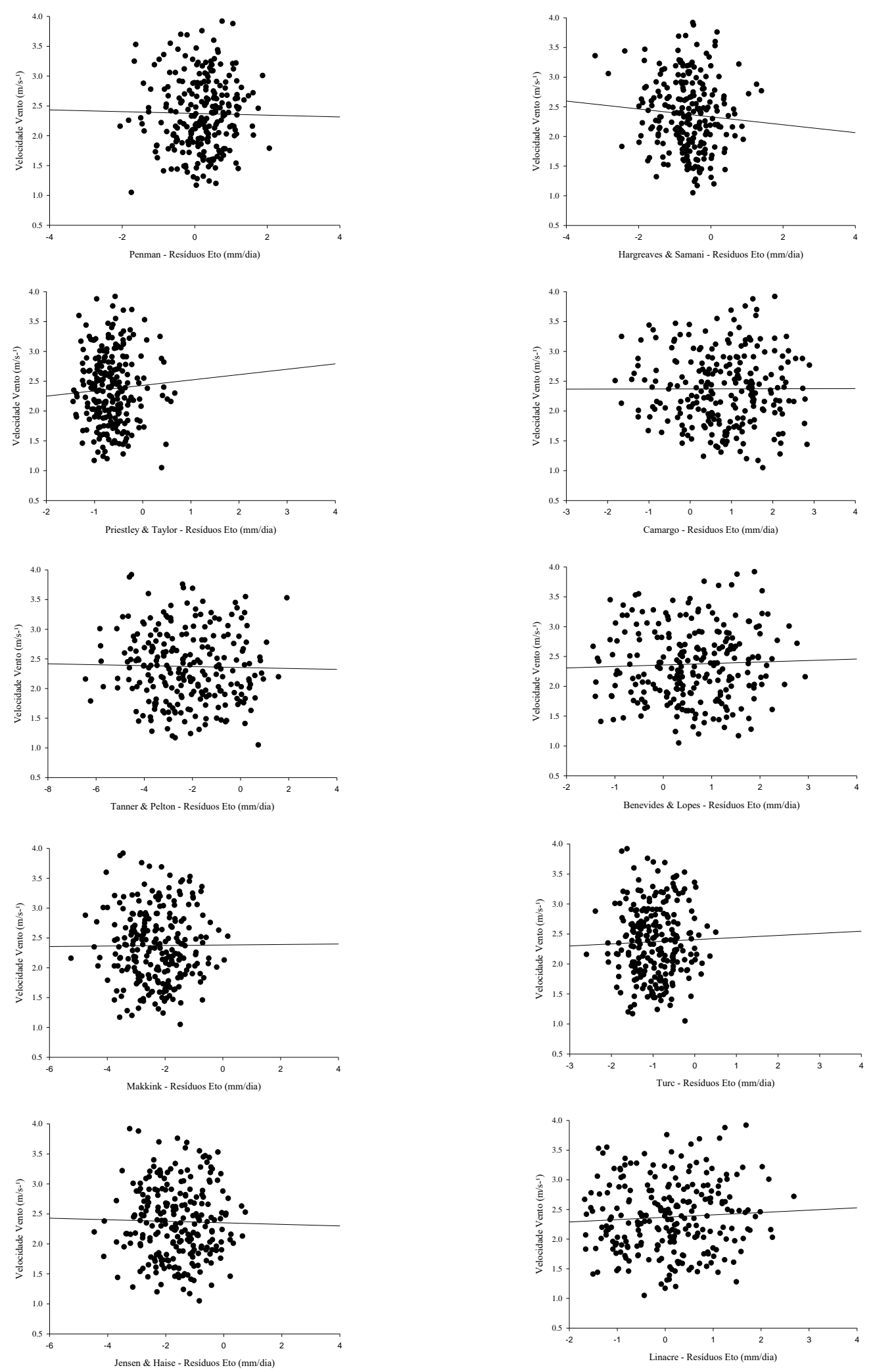

Figura 8. Resíduos da relação entre a variável velocidade do vento (u2) e sua influência nos métodos de determinação da ETo. Residues of the relationship between wind speed variable (u2) and its influence on ETo determination methods.

Fonte: Autoria própria. Own authorship. 
Para os demais métodos, observa-se variação na acurácia das estimativas em função da demanda evaporativa da atmosfera, sendo nesse trabalho, considerada alta quando Tmed $>$ média, URmin < 45\%, Q* > média ou u2 $>3 \mathrm{~m} \mathrm{~s}^{-1}$ e baixa quando Tmed < média, URmin > $45 \%$, Q* $<$ média ou u2 $<3 \mathrm{~m} \mathrm{~s}^{-1}$. Moeletsi et al. (2013) comparando o método de Hargreaves \& Samani com o método de Penman-Monteith para o sul da África, encontraram valores de ETo subestimados (outubro a maio) e superestimados (agosto e setembro) em diferentes épocas do ano, dependendo da condição atmosférica. No entanto, os autores concluem que o método apresenta tendência em subestimar os valores da ETo, corroborando com os resultados do presente trabalho, em que as estimativas da ETo pelo método de Hargreaves \& Samani apresentam valores de b menores que 1 em todas as condições, exceto para quando $\mathrm{Q}^{*}<$ média, quando observa-se uma leve superestimava $(1,041)$.

Anyadike (1987), estudando a precisão das estimativas do método de Linacre, concluiu que ele possui algumas características do método de Penman, mas que no entanto, em climas tropicais chuvosos geralmente resulta em estimativas subestimadas. Isso se deve-se ao fato de que o método não considera a umidade relativa propriamente, mas apenas a temperatura do ponto de orvalho, que em climas úmidos tende a ser menor, subestimando os valores de ETo em condições de umidade relativa alta, o que pode ser observado no coeficiente de regressão (Tabela 3 e Figura 4).

Longobardi e Villani (2013), estudando os métodos Penman-Monteith e Priestley \& Taylor para a região sul da Itália, encontraram valores anuais de ETo de 921 e $864 \mathrm{~mm}$ anuais, respectivamente; o método de Priestley \& Taylor subestimou Penman-Monteith no inverno, verão e outono e superestimou na primavera. Nesse estudo, o método de Priestley \& Taylor subestimou os valores de ETo, tendo os melhores resultados nos dias com URmin < 45\% (Figura 4 e Tabela 3). Esses resultados vão ao encontro com dos constatados por Minacapilli et al. (2016), que estudando métodos de estimativa da ETo em clima mediterrâneo típico no período de maio a agosto (verão), quando a umidade relativa do ar é baixa, encontraram estimativas satisfatórias utilizando os métodos de Priestley \& Taylor e Makkink. Por outro lado, para o presente estudo, o método de Makkink não foi eficiente, muito pelo fato de o ajuste ter sido efetivado para condições de clima árido, resultando em resíduos negativos e superestimação em todas as condições avaliadas.

Melo e Fernandes (2012) também utilizaram o método Jensen \&Haise para regiões áridas e semiáridas e obtiveram o mesmo padrão de estimativas de Makkink, com superestimativa de ETo, principalmente em condições de umidade relativa baixa (Figura 4), Q* > média (Tabela 4) e Tmed > média (Tabela 5).

O método de Turc esteve entre os cinco melhores métodos em todo o período, com RMSE de 1,092 (Tabela 2), superestimando a ETo em 20\%. No entanto, quando em condições de clima úmido, URmin > 45\% observou-se uma pequena melhoria nas estimativas, embasado pela diminuição no RMSE (Tabela 3) e pela tendência de resíduos próximos a 0, com o aumento da URmin (Figura 4). Esses resultados estão de acordo com os resultados de Martinez e Thepadia (2010), que analisando o método em uma região de clima úmido (Flórida, EUA) afirmam que o método apresentou as melhores estimativas, quando 
comparado ao método de Hargreaves \& Samani, não apresentando valores sub ou superestimados.

Para Tanner \& Pelton, constatou-se diminuição no RMSE de 3,576 mm (Q* média) para 1,504 mm ( $\mathrm{Q}^{*}<$ média), em concordância com os resultados de Caporusso e Rolim (2015), que o identificaram como o método mais preciso para a estação de inverno (período de baixa incidência de radiação) na região de Jaboticabal (São Paulo). Por outro lado, os resultados de Caporusso e Rolim (2015) apontam o método de Benevides \& Lopes como adequado para o verão. No entanto, nessa presente pesquisa é observado apenas uma pequena diminuição no valor de RMSE, quando da ocorrência de Tmed > média (Tabela 5), sendo observado o oposto para $\mathrm{Q}^{*}>$ média e URmin $<45 \%$.

O método de Camargo mostrou-se mais eficiente em condições de baixa demanda evaporativa, chegando a alcançar RMSE de $0,748 \mathrm{~mm}$ para $\mathrm{Q}^{*}<$ média e $0,908 \mathrm{~mm}$ para URmin > 45\%, não apresentando diferença significativa de Penman-Monteith pelo teste $\mathrm{t}$ student nestas condições. Essa melhora indica que o método é adequado para uma faixa de temperatura (Tmed) onde $Q^{*}$ e URmin são baixas e altas, respectivamente, uma vez que o

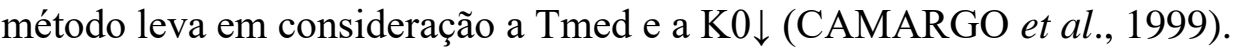

\section{CONCLUSÃO}

Os resultados expressos a partir da análise de correlação de Pearson e componentes principais evidenciaram de forma adequada os reais efeitos das variáveis climatológicas sobre os métodos de estimativa testados, apresentando baixos índices de correlação para a variável de velocidade do vento, e correlações positivas para temperatura e balanço de radiação e negativas para umidade relativa. Apesar de ser constatado as variações nas estimativas de ETo para os métodos avaliados quanto a seus resíduos, a análise de componentes principais apresentou alta explicação da variância dos dados.

Os resultados mostram que o efeito da radiação solar $\left(Q^{*}\right)$ tem maior impacto sobre os métodos de estimativas de ETo, evidenciando assim como a variável climatológica de maior influência no processo.

É recomendável a utilização do método padrão Penman-Monteith, porém, quando houver a ausência de variáveis, recomenda-se a utilização dos métodos de Linacre (Q*) e Hargreaves \& Samani ( $Q^{*}$ e umidade relativa). Métodos como o de Penman e Priestley \& Taylor atingiram boas estimativas, mas devido ao fato de utilizarem as mesmas variáveis do método padrão, exceto u2 para Priestley \& Taylor, recomenda-se a utilização do próprio método de Penman-Monteith.

\section{REFERÊNCIAS BIBLIOGRÁFICAS}

ALLEN, R. G.; PEREIRA, L. S.; RAES, D.; SMITH, M. Crop evapotranspiration: Guidelines for computing crop water requirements. Roma: FAO, 1998. 300 p. (Irrigation and Drainage Paper, 56). 
AHMED, S.; HUSSEIN, A. Grass ET estimates using Penman-type equations in Central Sudan. Journal of Irrigation and Drainage Engineering, New York, v. 125, n. 6, p.321329, 1999.

AZEVEDO, B. M.; ANDRADE, E. M.; SILVEIRA, S. S. Investigação da estrutura multivariada da evapotranspiração na região centro sul do Ceará pela análise de componentes principais. Revista Brasileira de Recursos Hídricos, Porto Alegre, v. 8, n. 1, p.39-44, 2003.

ANYADIKE, R. N. C. The Linacre evaporation formula tested and compared to others in various climates over west Africa. Agricultural and Forest Meteorology, Amsterdam, v. 39, n. 1, p.111-119, 1987.

BENEVIDES, J. G.; LOPEZ DIAZ, J. Formula para el calculo de la evapotranspiracion potencial adaptada al tropico $\left(15^{\circ} \mathrm{N}-15^{\circ} \mathrm{S}\right)$. Agronomia Tropical, Maracay, v. 20, n. 5, p.335-345, 1970.

BERENGENA, J., GAVILAN, P. Reference evapotranspiration estimation in a highly advective semiarid environment. Journal of Irrigation and Drainage Engineering, New York, v. 131, n. 2, p. 147-163, 2005.

BORGES JÚNIOR, J. C. F.; ANJOS, R. J.; SILVA, T. J. A.; LIMA, J. R. S.; ANDRADE, C. L. T. Métodos de estimativa da evapotranspiração de referência diária para a microrregião de Garanhuns, PE. Revista Brasileira de Engenharia Agrícola e Ambiental, Campina Grande, v. 16, n. 4, p.380-390, 2012.

ČADRO, S., UZUNOVIĆ M., ŽUROVEC J. Validation and calibration of various reference evapotranspiration alternative methods under the climate conditions of Bosnia and Herzegovina. International Soil and Water Conservation Research, Amsterdam, v. 5, n. 4, p.309-324, 2017.

CAMARGO, A. P.; MARIN, F. R.; SENTELHAS, P. C.; PICINI, A. G. Ajuste da equação de Thornthwaite para estimar a evapotranspiração potencial em climas áridos e super úmidos, com base na amplitude térmica diária. Revista Brasileira de Agrometeorologia, Santa Maria, v. 7, n. 2, p.251-257, 1999.

CAPORUSSO, N. B.; ROLIM, G. S. Reference evapotranspiration models using different time scales in the Jaboticabal region of São Paulo, Brazil. Acta Scientiarum Agronomy, Maringá, v. 37, n. 1, p.1-9, 2015.

CONCEIÇÃO, M. A. F. Estimativa da evapotranspiração de referência com base na temperatura do ar para as condições do Baixo Rio Grande, SP. Revista Brasileira de Agrometeorologia, Santa Maria, v. 11, n. 2, p.229-236, 2003.

FERNANDES, D. S.; HEINEMANN, A. B.; PAZ, R. L.; AMORIM, A. O. Evapotranspiração: uma revisão sobre os métodos empíricos. Santo Antônio de Goiás: Embrapa Arroz e Feijão, Ministério da Agricultura, Pecuária e Abastecimento, 2010. 44 p. (Documentos, 263).

HAIR, J. F.; ANDERSON, R. E.; TATHAM, R. L.; BLACK, W. C. Análise multivariada de dados. Porto Alegre: Bookman, 2005. 688 p. 
HALLAL, M. O. C.; SCHÖFFEL, E. R.; BRIXNER, E. R.; CUNHA, A. R. Métodos de estimativa da evapotranspiração de referência para Pelotas, Rio Grande do Sul. Amazonian Journal of Agricultural and Enviromental Science, Belém, v. 60, n. 1 , p.1-10, 2017.

JENSEN, M. E.; HAISE, H. R. Estimating evapotranspiration from solar radiation. Journal of Irrigation Drainage Division, New York, v. 89, n. 4, p.15-41, 1963.

KUINCHTNER, A.; BURIOL, G. A. Clima do estado do Rio Grande do Sul segundo a classificação climática de Köppen e Thornthwaite. Ciências Exatas, Rio de Janeiro, v. 2, n. 1, p.171-182, 2001.

LEMOS FILHO, L. C. A.; CARVAlHO, L. G.; EVANGElistA, A. W. P.; ALVES JÚNIOR, J. Análise espacial da influência dos elementos meteorológicos sobre a evapotranspiração de referência em Minas Gerais. Revista Brasileira de Engenharia Agrícola e Ambiental, Campina Grande, v. 14, n. 12, p.1294-1303, 2010.

LI, S.; KANG, S.; ZHANG, L.; ZHANG, J. On the attribution of changing crop evapotranspiration in arid regions using four methods. Journal of Hydrology, Amsterdam, v. 563, n. 1, p.576-585, 2018.

LIMA JÚNIOR, J. C.; ARRAES, F. D. D.; OLIVEIRA, J. B.; NASCIMENTO, F. A. L.; MACÊDO, K. G. Parametrização da equação de Hargreaves e Samani para estimativa da evapotranspiração de referência no Estado do Ceará, Brasil. Revista Ciência Agronômica, Jaboticabal, v. 47, n. 3, p.447-454, 2016.

LONGOBARDI, A.; VILLANI, P. The use of micrometeorological data to identify significant variables in evapotranspiration modeling. Procedia Environmental Sciences, Temerin, v. 19, n. 1, p.267-274, 2013.

MARTINEZ, C. J.; THEPADIA, M. Estimating reference evapotranspiration with minimum data in Florida. Journal of Irrigation and Drainage Engineering, New York, v. 136, n. 7, p.494-501, 2010.

MCVICAR, T. R; RODERICK, M. L.; DONOHUE, R. J.; LI, L. T.; VAN NIEL, T. G.; THOMAS, A.; GRIESER, J.;JHAJHARIA, D.; HIMRI, Y.; MAHOWALD, N. M.; MESCHERSKAYA, A. V.; KRUGER, A. C.; REHMAN, S.; DINPASHOH, Y. Global review and synthesis of trends in observed terrestrial near surface wind speeds: Implications for evaporation. Journal of Hydrology, Amsterdam, v. 416-417, n. 1, p. 182-205, 2012.

MELO, G. L.; FERNANDES, A. L. T. Evaluation of empirical methods to estimate reference evapotranspiration in Uberaba, state of Minas Gerais, Brazil. Engenharia Agrícola, Jaboticabal, v. 32, n. 5, p.875-888, 2012.

MINACAPILLI, M.; CAMMALLERI, C.; CIRAOLO, G.; RALLO, G.; PROVENZANO, G. Using scintillometry to assess reference evapotranspiration methods and their impact on the water balance of olive groves. Agricultural Water Management, Amsterdam, v. 170, n. 1, p.49-60, 2016.

MOELETSI, M. A.; WALKER, S.; HAMANDAWANA, H. Comparison of the Hargreaves and Samani equation and the Thornthwaite equation for estimating dekadal evapotranspiration 
in the Free State Province, South Africa. Physics and Chemistry of the Earth, Oxford, v. 66, n. 1, p.4-15, 2013.

PANDEY, P. K.; DABRAL, P.P.; PANDEY, V. Evaluation of reference evapotranspiration methods for the northeastern region of India. International Soil and Water Conservation Research, Amsterdam, v. 4, n. 1, p.52-63, 2016.

PEREIRA, D. R.; YANAGE, S. N. M.; MEllO, C. R.; SIlVA, A. M.; SIlVA, L. A. Desempenho de métodos de estimativa da evapotranspiração de referência para a região da Serra da Mantiqueira, MG. Ciência Rural, Santa Maria, v. 39, n. 9, p.2488-2493, 2009.

SHARMA, M. L. Estimating Evapotranspiration. In: HILLEL, D. (ed.). Advances in Irrigation. New York: Academic, 1985. v. 3, p. 213-281

SILVA, V. J.; CARVAlHO, H. P.; SILVA, C. R.; CAMARGO, R.; TEODORO, R. E. F. Desempenho de diferentes métodos de estimativa da evapotranspiração de referência diária em Uberlândia, MG. Bioscience Journal, Uberlândia, v. 27, n. 1, p.95-101, 2011.

STATSOFT. Programa computacional Statistica 7.0. E.A.U. 2004.

STOY, P. C.; KATUl, G. G.; SIQUEIRA, M.; JUANG, J. H.; NOVICK, K. A.; McCARTHY, H. R.; OISHI, A. C.; UEBELHERR, J. M.; KIM, H. S.; OREN, R. Separating the effects of climate and vegetation on evapotranspiration along a successional chronosequence in the southeastern US. Global Change Biology, Oxford, v. 12, n. 11, p.2115-2135, 2006.

TABARI, H.; GRISMER, M. E.; TRAJKOVIC, S. Comparative analysis of 31 references evapotranspiration methods under humid conditions. Irrigation Science, New York, v. 31, n. 1, p.107-117, 2011.

TEMESGEN, B.; ALLEN, R.; JENSEN, D. Adjusting temperature parameters to reflect wellwater conditions. Journal of Irrigation and Drainage Engineering, New York, v. 125, n. 5, p.26-33, 1999.

TURC, L. Estimation of irrigation water requirements, potential evapotranspiration: a simple climatic formula evolved up to date. Annals of Agronomy, Madison v. 12, n. 1, p.13-49, 1961.

VEGA, E. C.; JARA, J. C. Estimación de la evapotranspiración de referência para dus zonas (Costa y Región Andina) del Ecuador. Engenharia Agrícola, Jaboticabal, v. 29, n. 3, p.390403, 2009.

WIDMOSER, P. A discussion on and alternative to Penman-Monteith equation. Agricultural Water Management, Amsterdam, v. 96, n. 4, p.711-721, 2009.

YU, X.; LAMAČOVÁ, A.; DUFFY, C.; KRÁM, P.; HRUŠKA, J. Hydrological model uncertainty due to spatial evapotranspiration estimation methods. Computer \& Geosciences, Quebec, v. 90, n. 2, p.90-101, 2016. 